\title{
Observation of Fluorescence of the $\mathrm{HeH}$ Molecule
}

\author{
Thomas Möller, Michael Beland, and Georg Zimmerer \\ II. Institut für Experimentalphysik der Universität Hamburg, D-2000 Hamburg 50, Federal Republic of Germany
}

(Received 8 July 1985)

\begin{abstract}
This Letter reports the first spectroscopic detection of $\mathrm{HeH}$, the simplest excimer molecule that can exist. $\mathrm{HeH}^{*}$ is produced in electronically excited states in reactions between $\mathrm{He}$ and $\mathrm{H}_{2}$ excited selectively by synchrotron radiation into the $C^{1} \Pi_{\mathrm{u}}\left(v^{\prime} \geqslant 1\right)$ or $B^{1} \Sigma_{\mathrm{u}}^{+}\left(v^{\prime} \geqslant 11\right)$ state. $\mathrm{HeH}^{*}$ emits a broad characteristic fluorescence continuum between 200 and $400 \mathrm{~nm}$ which is attributed to $B^{2} \Pi \rightarrow X^{2} \Sigma^{+}$.

PACS numbers: $33.20 . \mathrm{Lg}, 33.50 .-\mathrm{j}, 34.50 .-\mathrm{s}$
\end{abstract}

$\mathrm{HeH}$ is one of the smallest molecular systems and has attracted theoretical interest for a long time. ${ }^{1,2}$ Its ground state is strongly repulsive; however, strongly bound excited states were predicted by a molecularorbital calculation twenty years ago. ${ }^{2}$ The main contribution to the bond is the polarization of the $\mathrm{He}$ atom by the $\mathrm{H}^{+}$core if $\mathrm{H}$ penetrates the electronic cloud of He. For the lowest excited states, $A^{2} \Sigma^{+}$and $B^{2} \Pi$, a binding energy of $\sim 2 \mathrm{eV}$ was predicted. ${ }^{2}$ Recently, more sophisticated $a b$ initio calculations have underlined the existence of bound excited states. ${ }^{1}$

To the best of our knowledge, up to now no spectroscopic evidence of bound excited states of $\mathrm{HeH}$ has been found. The same is true for the other rare-gas hydrides with the only exception being ArH. Johns ${ }^{3}$ reported infrared fluorescence bands in a gas discharge which he ascribed to a ${ }^{2} \Pi \rightarrow{ }^{2} \Sigma^{+}$transition of $\mathrm{ArH}$. Very recently, Sadeghi et al. ${ }^{4}$ assigned a fluorescence continuum in the uv region which is generated in reactive collisions of thermal $\mathrm{Ar}$ metastables with $\mathrm{H}_{2}$ to ArH emission.

In this paper we report on the first spectroscopic proof of the existence of rather long-lived excited states of $\mathrm{HeH}$. They lead to broad uv bound-free fluorescence continua terminating at $X^{2} \Sigma^{+}$. Similar results were also obtained for the other rare-gas hydrides. ${ }^{5}$ Here we concentrate on $\mathrm{HeH}$, which is most important from the theoretical point of view.

Electronically excited $\mathrm{HeH}$ molecules were obtained from elementary reactions of the type

$$
\mathrm{He}+\mathrm{H}_{2}^{*} \rightarrow \mathrm{HeH}^{*}+\mathrm{H}
$$

in $\mathrm{H}_{2}$-doped $\mathrm{He}$. The pressures ranged between 0 and 10 Torr $\mathrm{H}_{2}$ and 1 and 20 Torr He. Primary excitation of $\mathrm{H}_{2}$ was performed optically with synchrotron radiation on the SUPERLUMI beam line at HASYLAB. ${ }^{6,7}$

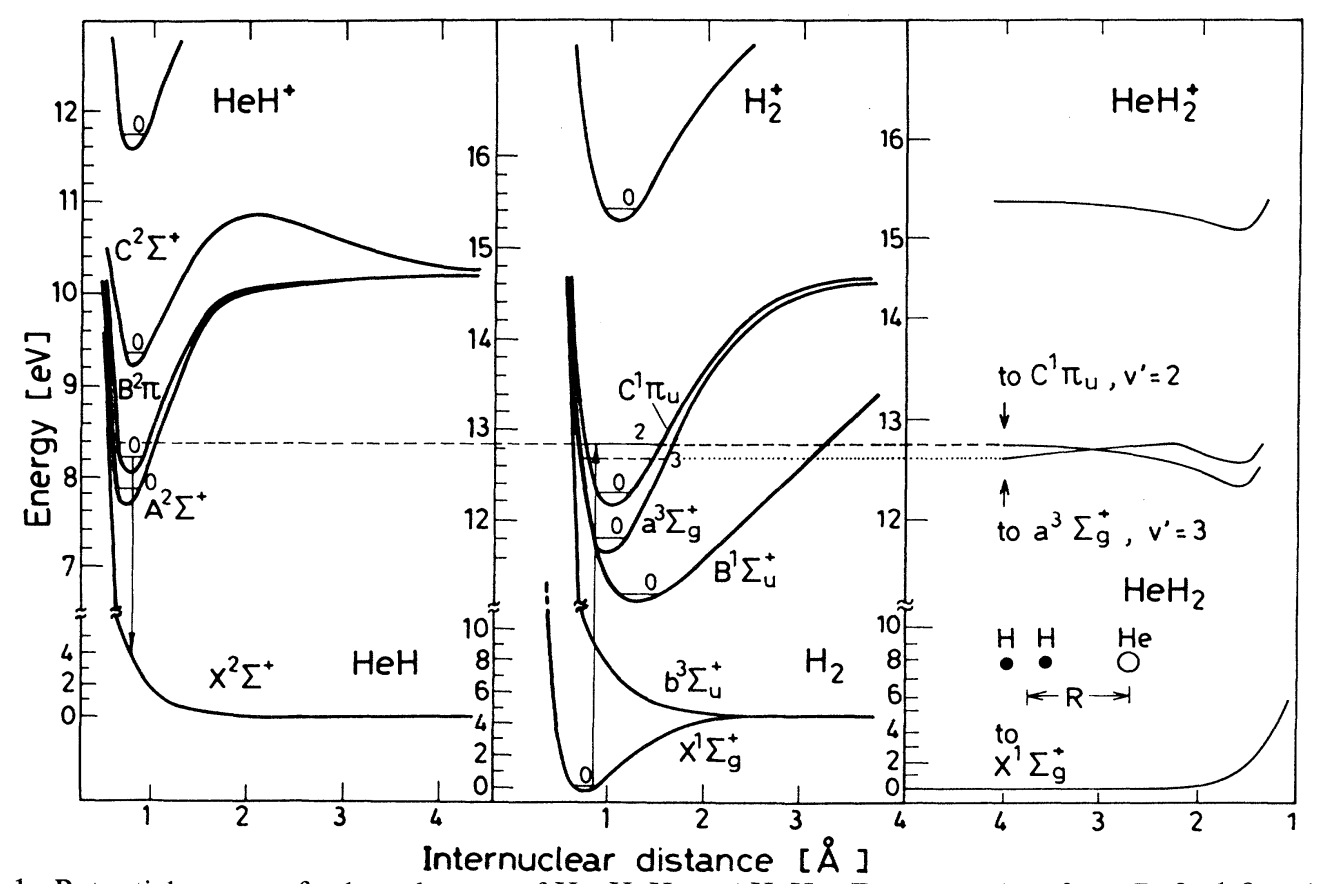

FIG. 1. Potential curves of selected states of $\mathrm{H}_{2}, \mathrm{HeH}$, and $\mathrm{HeH}_{2}$. Data are taken from Refs. 1,8 and 9. 


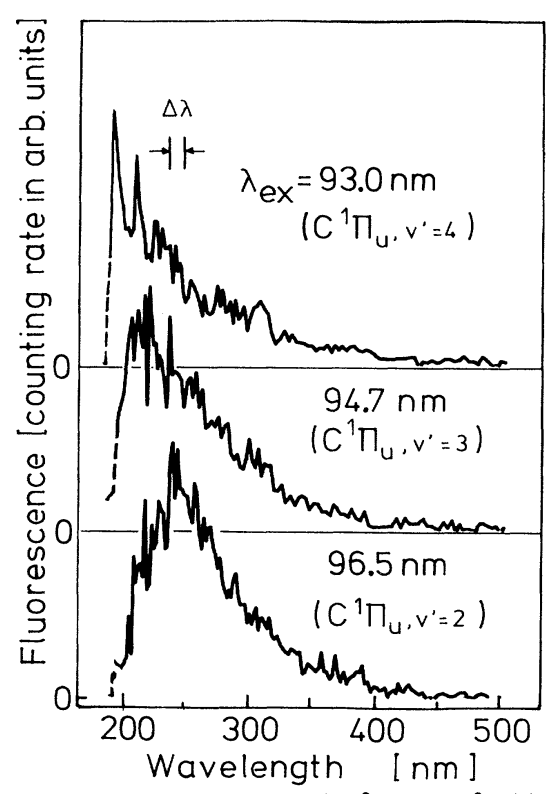

FIG. 2. Fluorescence of $\mathrm{HeH}\left(B^{2} \Pi \rightarrow X^{2} \Sigma^{+}\right)$for differnt excitations into $\mathrm{H}_{2} C^{1} \Pi_{\mathrm{u}}$. The spectra are corrected for the spectral response of the system; below $200 \mathrm{~nm}$ they are uncertain because of the cutoff of the response function.

The spectral resolution was sufficiently high for rotationally selective excitation of $\mathrm{H}_{2}$.

Figure 1 shows the calculated potential curves of $\mathrm{HeH}^{1}$ and those $\mathrm{H}_{2}$ potential curves ${ }^{8}$ which are important for this paper. If we assume optical excitation, it immediately follows from Fig. 1 that $\mathrm{H}_{2}$ has to be excited either into high vibrational levels of the $B$ state or into the $C$ state. Therefore, excitation wavelengths below the LiF cutoff are required. For this reason, the gas cell used was equipped with a thin In window (thickness $100 \mathrm{~nm}$ ).

The fluorescence light was analyzed perpendicular to the exciting beam with a band pass of $10 \mathrm{~nm}$. The signal-to-noise ratio was improved substantially by exploitation of the time structure of synchrotron radiation. Fluorescence was recorded only within a short time window $(\sim 10 \mathrm{~ns})$ immediately following the excitation pulses $(\mathrm{FWHM} \sim 150 \mathrm{ps}$, repetition rate 1 $\mathrm{MHz}$ ). The length of the time window roughly corresponds to the lifetime of the emitting state.

Fluorescence spectra which are observed following selective excitation of $v^{\prime}=2,3$, and 4 of the $C^{1} \Pi_{u}$ state of $\mathrm{H}_{2}$ in a mixture of 0.3 Torr $\mathrm{H}_{2}$ and 20 Torr He are shown in Fig. 2. For the lowest excitation energy, a broad, asymmetric continuum is observed with a maximum at $235 \mathrm{~nm}$. With increasing excitation energy, the continuum further broadens and shifts to shorter wavelengths. For $v^{\prime}=4$ excitation it already reaches the cutoff of the transmission of the monochromator (dashed part of the curves).

The observed spectra are ascribed to the decay of $\mathrm{HeH}$ created via (1). Between the two $\mathrm{HeH}$ excited states $\left(A^{2} \Sigma^{+}\right.$and $\left.B^{2} \Pi\right)$ which have to be taken into consideration as emitting states (see Fig. 1), we prefer to assign the emission to $B^{2} \Pi \rightarrow X^{2} \Sigma^{+}$transitions. Johns ${ }^{3}$ showed that the $A^{2} \Sigma^{+}$state of ArH strongly predissociates. Because of the great similarities among the rare-gas hydride electronic structure, the same may hold also for $\mathrm{HeH}$.

It must be pointed out clearly that in the wavelength

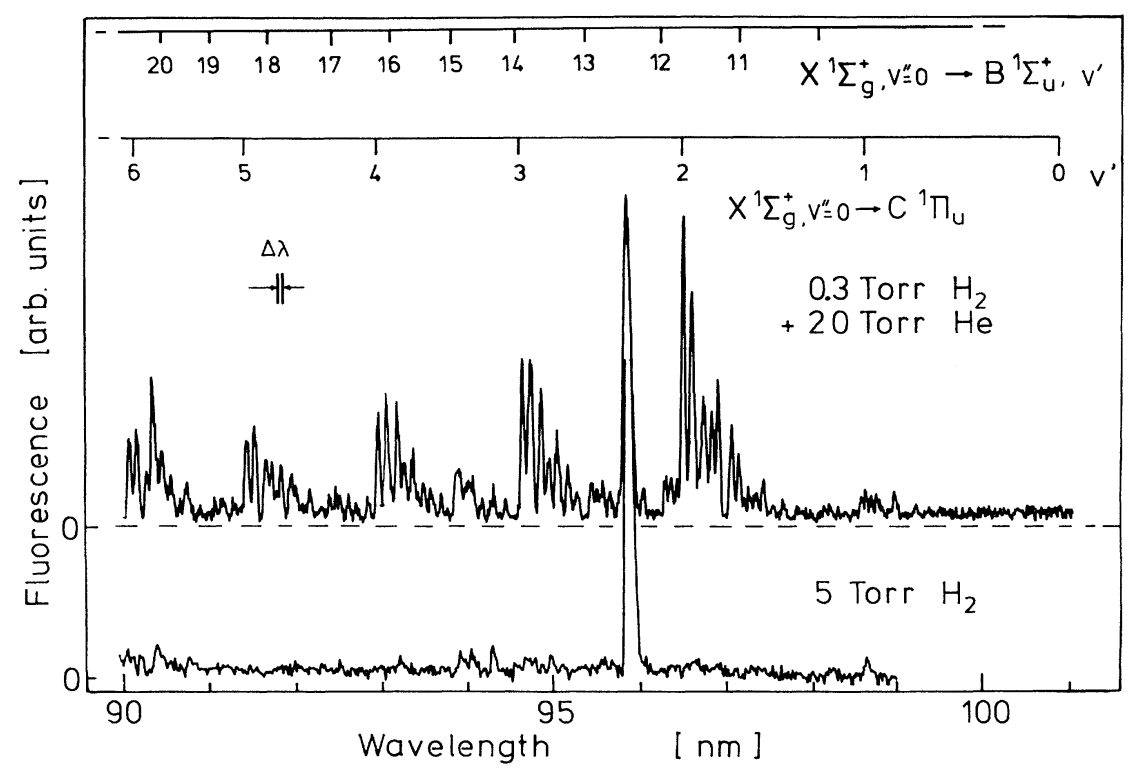

FIG. 3. Excitation spectrum of near-uv fluorescence $\left(210-350 \mathrm{~nm}\right.$ ) of pure $\mathrm{H}_{2}$ (5 Torr) and a mixture of 0.3 Torr $\mathrm{H}_{2}$ and 20 Torr He. 
range of the observed emission, in, e.g., gas discharges, the $a^{3} \Sigma_{\mathrm{g}}^{+} \rightarrow b^{3} \Sigma_{\mathrm{u}}^{+}$continuum of $\mathrm{H}_{2}$ is also emitted. ${ }^{10}$ Though optical excitation of $\mathrm{H}_{2}$ in the $\mathrm{H}_{2}$ He mixtures terminates at ungerade singlet states, one has to take into consideration collision-induced intersystem crossing (ISC), leading to population of the $\mathrm{H}_{2} a^{3} \mathrm{\Sigma}_{\mathrm{g}}^{+}$state and its subsequent emission. The results presented now strongly support our assignment of the observed fluorescence.

In Fig. 3 we present excitation spectra of the fluorescence emitted in the wavelength interval $210-350 \mathrm{~nm}$. The lower curve was obtained from 5 Torr $\mathrm{H}_{2}$ without He. All the observed lines can be ascribed to $\mathrm{N}_{2}$ impurities. Though the contamination of $\mathrm{H}_{2}$ by $\mathrm{N}_{2}$ was small $(<200 \mathrm{ppm})$, the $\mathrm{N}_{2}$ excitation lines (e.g., at $95.8 \mathrm{~nm}$ ) show up because they lead to the allowed $C^{\prime 1} \Sigma_{\mathrm{u}}^{+} \rightarrow a^{1} \Sigma_{\mathrm{g}}^{+}$and $b^{1} \Pi_{\mathrm{u}} \rightarrow a^{1} \Sigma_{\mathrm{g}}^{+}$transitions which emit within the wavelength interval of observation. ${ }^{11}$ Following primary excitation of $\mathrm{H}_{2}$ itself, no emission between 210 and $350 \mathrm{~nm}$ was observed. This shows that, even at the relatively high gas pressure used, in pure $\mathrm{H}_{2}$ the collision-induced ISC can be neglected.

In the presence of $\mathrm{He}$ (upper curve; $20 \mathrm{Torr} \mathrm{He}+$ 0.3 Torr $\mathrm{H}_{2}$ ) clearly the rotational lines of the $C^{1} \Pi_{\mathrm{u}}$ state $\left(v^{\prime}=2,3,4,5 ; v^{\prime}=1\right.$ very weak) are resolved. No signal is observed under $v^{\prime}=0$ excitation. In addition to the $C$ state, rotational lines of the $B$ state $\left(v^{\prime} \geqslant 11\right)$ are observed, with smaller intensity, however. Various band heads of transitions to the $B$ and the $C$ states are marked in Fig. $3 .^{8}$

The excitation spectrum clearly yields a threshold for the population of the emitting state. In the crudest approximation one might take the energy of $v^{\prime}=1$ of the $\mathrm{H}_{2} \mathrm{C}$ state as the threshold energy. It correlates well with the theoretical prediction for the energetic threshold of the elementary reaction (1) which can be deduced from the calculated potential curves. ${ }^{1}$ This is a strong support for our assignment of the observed emission. The other rare-gas $-\mathrm{H}_{2}$ systems also all have a clear onset of the rare-gas hydride emission. ${ }^{5}$ The individual onsets, however, are markedly different in agreement with the different binding energies of the emitting states involved. For the $\mathrm{H}_{2}$-Ar system, we wish to point out that the threshold of the emission ascribed to the ArH molecule is significantly below the minimum of the $\mathrm{H}_{2} a^{3} \Sigma_{\mathrm{g}}^{+}, 5$ in agreement with the data of Sadeghi et al. ${ }^{4}$ From the observed onsets and the well-known dissociation limits of the rare-gas hydride $B^{2} \Pi$ states $\left(\mathrm{He}^{1} S+\mathrm{H}^{* 2} P\right)$, the binding energies of the $B$ states can be estimated. Results are given in Table I and compared with theoretical predictions. Two values for the well depth are available for $\mathrm{HeH}$. The experimental value for the dissociation energy of the $B$ state is in good agreement.

Now we may comment on the spectral distribution of $\mathrm{HeH}$ emission for different excitation energies (Fig.
TABLE I. Dissociation energies $\left(D_{0}\right)$ of rare-gas hydride $B^{2} \Pi$ states [terminating at $\left.\mathrm{H}^{*}\left({ }^{2} P\right)\right]$ in comparison with calculated binding energies $\left(D_{e}\right)$.

\begin{tabular}{lcc}
\hline \hline & $\begin{array}{c}D_{0}(\mathrm{eV}) \\
\text { This work }\end{array}$ & $\begin{array}{c}D_{e}(\mathrm{eV}) \\
\text { Theory }\end{array}$ \\
\hline $\mathrm{HeH}$ & 2.05 & $2.20^{\mathrm{a}}$ \\
$\mathrm{NeH}$ & 1.80 & $1.94^{\mathrm{b}}$ \\
$\mathrm{ArH}$ & 2.93 & $1.67^{\mathrm{a}}$ \\
$\mathrm{KrH}$ & 2.9 & $2.75^{\mathrm{a}}$ \\
$\mathrm{XeH}$ & $\geqslant 3.6$ & \\
\hline \hline
\end{tabular}

aFrom Ref. 1.

bFrom Ref. 2.

2). Following $\mathrm{H}_{2} \mathrm{C}\left(\boldsymbol{v}^{\prime}=2\right)$ excitation near to the threshold, in the subsequent elementary reaction (1) predominantly $\mathrm{HeH} B\left(v^{\prime}=0\right)$ molecules are produced. The decay of such vibrationally relaxed molecules into a strongly repulsive final state leads to a broad, single-centered band in agreement with observation. With increasing excitation energy, higher vibrational levels of the $\mathrm{HeH} B$ state may be populated. This explains the observed broadening. The shift to shorter wavelengths is ascribed to an effect of FranckCondon factors and the $\nu^{3}$ dependence of the transition probabilities. We should also mention that fluorescence from the energetically nearest level $v^{\prime}=3$ of the $\mathrm{H}_{2} a^{3} \mathrm{\Sigma}_{\mathrm{g}}^{+}$state looks completely different. ${ }^{10}$

We also measured fluorescence spectra of 10 Torr He doped with 0.3 Torr $\mathrm{H}_{2}$ including the vacuum-uv spectral range. Bound-bound $\mathrm{H}_{2} \mathrm{C} \rightarrow X$ transitions around $120 \mathrm{~nm}$ and bound-free fluorescence of the $\mathrm{H}_{2}$ $B$ state around $160 \mathrm{~nm}$, emitted at large internuclear distances, ${ }^{12}$ and the broad continuum of $\mathrm{HeH}$ at 235 nm were observed.

The intensity ratio of $\mathrm{H}_{2}$ and $\mathrm{HeH}$ fluorescence gives a rough estimate of the total cross section, $\sigma_{f}$, of the reaction

$$
\mathrm{H}_{2} C^{1} \Pi_{\mathrm{u}}\left(v^{\prime}=2\right)+\mathrm{He} \rightarrow \mathrm{HeH} B^{2} \Pi+\mathrm{H}
$$

The Franck-Condon factors of $\mathrm{H}_{2} \mathrm{C} \rightarrow X$ and $B \rightarrow X$ transitions are known well enough ${ }^{13}$ to calculate the total $\mathrm{H}_{2}$ fluorescence intensity from the observed part of the spectrum. With neglect of additional quenching of $\mathrm{H}_{2}$ and $\mathrm{HeH}$ fluorescence (which is justified in view of the low gas pressures used), the ratio of the fluorescence intensities is then given by

$$
I_{\mathrm{HeH}} / I_{\mathrm{H}_{2}}=\Gamma_{f} / \Gamma_{\mathrm{rad}} \text {. }
$$

$\Gamma_{f}$ is the rate of formation of $\mathrm{HeH}$, and $\Gamma_{\text {rad }}$ is the radiative decay rate of the primarily excited $\mathrm{H}_{2}$ molecule which was measured separately. $\Gamma_{f}$ obtained from (3) is finally converted into $\sigma_{f}$ with use of classical kinetic expressions. As a result, we obtain $\sigma_{f}=1.6 \pm 1 \AA^{2}$ for 
the $C\left(v^{\prime}=2\right)$ state. This is an average value concerning the rotational quantum number $\left(J^{\prime} \leqslant 3\right)$. Although the estimate of $\sigma_{f}$ is only approximate, the order of magnitude is important to rule out that the observed fluorescence around $235 \mathrm{~nm}$ is due to emission of the $\mathrm{H}_{2} a^{3} \Sigma_{\mathrm{g}}^{+}$state.

For $C\left(v^{\prime}=1\right)$, the cross section seems to be much smaller. This may be explained in the following way. A more rigorous evaluation of the threshold for the elementary reaction (2) has to take into account also endothermic reactions of particles in the tail of the Maxwellian velocity distribution, and would place the energetic threshold somewhere between $v^{\prime}=1$ and 2 . However, it is not the purpose of this paper to discuss these details.

Now we estimate the cross section for the reaction

$$
\mathrm{H}_{2} C^{1} \Pi_{\mathrm{u}}\left(v^{\prime}=2\right)+\mathrm{He} \rightarrow \mathrm{H}_{2} a^{3} \Sigma_{\mathrm{g}}^{+}+\mathrm{He} .
$$

From the $\mathrm{H}_{2}$ potential curves (Fig. 1) it follows that collision-induced ISC of $C\left(v^{\prime}=2\right)$ and $a\left({ }^{3} \Sigma_{\mathrm{g}}^{+}\right)$most probably populates $v^{\prime}=3$ of the $a$ state because of the small energetic distance. This is also reasonable in view of calculated potential surfaces of the $\mathrm{HD}+\mathrm{He}$ system. ${ }^{9}$ In their paper Römelt, Peyerimhoff, and Buenker present results for $\mathrm{He}+\mathrm{HD}\left(C^{1} \Pi_{\mathrm{u}}\right)$ and of $\mathrm{He}+\mathrm{HD}\left(a^{3} \Sigma_{\mathrm{g}}^{+}\right)$, both for $\boldsymbol{v}^{\prime}=0$ and different geometries. ${ }^{9}$ By adding the known vibrational energies, we obtain the corresponding curves for $v^{\prime}=2$ ( $C$ state) and $v^{\prime}=3$ ( $a$ state) shown in the right part of Fig. 1 (collinear geometry). They may be a good estimate for the $\mathrm{He}+\mathrm{H}_{2}$ system. The curves cross one another at a distance of about $3 \AA$ between the $\mathrm{He}$ atom and the center of $\mathrm{H}_{2}$.

During the collision, the $\mathrm{g} / \mathrm{u}$ symmetry of the dissociation limits is destroyed. The spin-orbit and spinspin interactions lead to a perturbation in the crossing region, and the resulting adiabatic potential curves yield an energy gap. As an upper limit, we tentatively took twice the spin-orbit interaction energy $E_{\text {s.o. }}=0.3$ $\mathrm{cm}^{-1}$, of the atoms. ${ }^{14}$

The probability for the $C \rightarrow a$ transition during one passage of the crossing region is estimated with the Landau-Zener probability ${ }^{15}$

$$
p=1-\exp \left\{-4 \pi^{2} \Delta E^{2} / h v|\Delta F|\right\} .
$$

$2 \Delta E$ is the width of the gap, $F$ is the difference of the gradients of the diabatic potential curves, and $v$ is the relative velocity of the particles in the crossing region. With $2 \Delta E=0.6 \quad \mathrm{~cm}^{-1}, \quad|\Delta F|=3 \times 10^{-10} \mathrm{~N}, \quad$ and $v=2.2 \times 10^{3} \mathrm{~ms}^{-1}$, we obtain $p=3.3 \times 10^{-6}$.

This value must be compared with the yet unknown probability, $p_{R}$, of reaction (2). As an estimate, we take the calculated probability of the reaction $\mathrm{H}_{2}^{+}+\mathrm{He} \rightarrow \mathrm{HeH}^{+}+\mathrm{H}$, which is about $10^{-1}$ to $5 \times 10^{-1} .{ }^{16}$ The result of Stroud et al. ${ }^{16}$ is valid for the direct reaction without a long-living intermediate collision complex. The potential surfaces of $\mathrm{H}_{2}(C)+\mathrm{He}$ and $\mathrm{H}_{2}^{+}+\mathrm{He}$ are very similar (see Fig. 1). Therefore, in the reaction of the neutrals, a long-living collision complex is not expected, either. It is obvious that the ISC probability cannot compete with the probability of the chemical reaction. It is interesting to note that the cross section for $\mathrm{H}_{2}^{+}+\mathrm{He} \rightarrow \mathrm{HeH}^{+}+\mathrm{H}, \sigma \approx 1 \AA^{2}, 17$ is similar to our result, $\sigma_{f}=1.6 \AA^{2}$, ascribed to $\mathrm{H}_{2}(C)+\mathrm{He} \rightarrow \mathrm{HeH}+\mathrm{H}$.

The authors are grateful for financial support from the Bundesminister für Forschung und Technologie of the Federal Republic of Germany.

${ }^{1} \mathrm{G}$. Theodorakopoulos, S. C. Farantos, R. J. Buenker, and S. D. Peyerimhoff, J. Phys. B 17, 1453 (1984).

${ }^{2}$ H. H. Michels and F. E. Harris, J. Chem. Phys. 39, 1464 (1963).

3J. W. C. Johns, J. Mol. Spectrosc. 36, 488 (1970).

${ }^{4}$ N. Sadeghi, T. D. Dreiling, H. Ben Kraiem, M. Touzeau, and R. De Souza, in Proceedings of the Thirty-Eighth International Meeting of the Society of Chemical Physics of France, Bombannes, France, 17-21 September 1984, Book of Abstracts (unpublished), p. 47.

${ }^{5}$ T. Möller, M. Beland, and G. Zimmerer, to be published.

${ }^{6} \mathrm{H}$. Wilcke, W. Böhmer, and N. Schwentner, Nucl. Instrum. Methods 208, 59 (1983).

${ }^{7}$ P. Gürtler, E. Roick, G. Zimmerer, and M. Pouey, Nucl. Instrum. Methods 208, 835 (1983).

${ }^{8}$ T. E. Sharp, At. Data 2, 119 (1971).

9J. Römelt, S. D. Peyerimhoff, and R. J. Buenker, Chem. Phys. 41, 133 (1979).

${ }^{10}$ H. M. James and A. S. Coolidge, Phys. Rev. 55, 184 (1939).

${ }^{11}$ A. Lofthus and P. H. Krupenie, J. Phys. Chem. Ref. Data 6, 113 (1977).

12A. Dalgarno, G. Herzberg, and T. L. Stephens, Astrophys. J. 162, L49 (1970).

13R. J. Spindler, J. Quant. Spectrosc. Radiat. Transfer 9, 627 (1969).

${ }^{14}$ C. E. Moore, Atomic Energy Levels as Derived from Analysis of Optical Spectra, National Bureau of Standards Circular No. 467 (U. S. GPO, Washington, D. C., 1949).

${ }^{15}$ E. E. Nikitin, Theory of Elementary Atomic and Molecular Processes in Gases (Clarendon, Oxford, 1974).

${ }^{16}$ C. Stroud, N. Sathyamurthy, R. Rangarajan, and L. M. Raff, Chem. Phys. Lett. 48, 350 (1977).

${ }^{17}$ T. Turner, O. Dutuit, and Y. T. Lee, J. Chem. Phys. 81, 3475 (1984). 